\title{
Virtual Simulation for Entertainment using Genetic Information
}

\author{
Jin Woo Kim ${ }^{1}$, Hun $\mathrm{Lim}^{2}$, Taeho You ${ }^{3}$, Mee Young Sung ${ }^{4} *$ \\ Department of Computer Science, Kent State University, Ohio, USA ${ }^{1}$ \\ Department of Computer Science and Engineering, Incheon National University, South Korea ${ }^{2,3,4}$
}

\begin{abstract}
The genetic information has been researched to predict the disease and to discover the clue of biological causes, especially in the medical field. However, based on the reliability of genetic information, it also gives a powerful realistic experience with VR devices. In this paper, we developed a dating simulation game that users can meet celebrities. The human personality of the celebrity, the favorability feedback depends on each choice of the conversation and a proper choice creation is based on the genetic information of the user and a celebrity. Besides, a method for utilizing genetic DNA information for virtual simulations is proposed. In this study, we have established that DNA information is related to human relationships both in the real and virtual worlds. Also, we concluded that the DNA information contributes to the development of interpersonal and mental health. However, a person's personality or preference for a specific situation or object, etc. are not determined only through genes. Therefore, more quantitative and multifaceted studies will need to be conducted on the effect of genes on personal preferences. We only experimented with virtual characters in virtual reality. It would be meaningful to proceed with the human experiment not only with a virtual character in the virtual environment, but also another human in the virtual environment. Eventually, the result of the experiment between virtual characters and humans should be compared.
\end{abstract}

Keywords-Virtual simulation; entertainment; genetic information; DeoxyriboNucleic Acid (DNA) matching; celebrity; favorability

\section{INTRODUCTION}

The research goal of this study is to validate how the similarity between the genetic information of a celebrity and that of a user influences the user's preference in virtual simulations for entertainment. To achieve this objective, we developed a dating simulation with celebrities utilizing DeoxyriboNucleic Acid (DNA) data. It also proposes a method to measure the similarity between the celebrity and the user's genetic DNA information so that the user experiences a strong intimacy with the celebrity.

By analyzing the DNA information, the constitution and personality of a celebrity can be grasped [1]. The genetic characteristics of a celebrity chosen by a user can allow the user to feel close to the celebrity and feel lively in Virtual Reality (VR) simulations.

Genetic information analysis companies, such as 23 andMe [2], Illumina [3], etc. try to collect genetic information and have focused on medical research and applications. To increase

*Corresponding Author

This work was supported by Incheon National University Research Grant in 2017 (No. 2017-0320). the speed of collecting genetic information, researches on entertainment using genetic information are needed to be accelerated.

It is also necessary to build a database by understanding the meaning of domestic genetic information and organizing the data to be extracted. Besides, the interaction between genetic data and new functionalities that may arise due to it should be studied. It also demands the analysis of the applications and related technologies concerning VR. To maximize the visual effects of VR, researches on 360-degree image rendering and shooting techniques are also needed.

Besides, researches on how to form an intimacy with celebrities are required. We research the psychological effects of users who are not intimate with celebrities through the relationship between the visual effects of VR and genetic information.

The rest of this paper proceeds with a presentation of the background including genetic information analysis and artificial reality/virtual reality/mixed reality (AR/VR/MR) simulations. Then, our system model's conceptual structure and DNA matching methods are described. Next, the details of the implementation of our prototype for experiments and the methods of experiments are explained. Lastly, our conclusion and future work are followed.

\section{BACKGROUND}

\section{A. Genetic Information Analysis}

Humans have 23 pairs of chromosomes including 22 pairs of autosomes and 1 pair of sex chromosomes. The 23andMe, an American personal genetic information analysis company, provides various benefits with consumer's genetic information by using a Direct-to-Consumer (DTC) method that sells directly to consumers without going through a doctor. With this genetic information, more than 250 types of analysis, including risk analysis for various diseases, sensitivity to drugs, genetic characteristics, and ancestor analysis, are becoming a boom by improving the quality of life of users [4]. Such attempts have also begun in Korea. Ewon Diagnostics Genome Center (EDGC) [5], a genome-specialized analysis company, started the DTC genetic test service under the official brand name Gene2Me [6] for the first time.

However, Korean genetic testing regulations are much stricter than in the US, so there is a limit to the market formation. This is because commercial genetic testing is considered ethical. As a result, it is not getting more users. 
Nevertheless, since June 2016, regulations have been easing, and as the number of users of genetic information increases, the importance of genetic information will emerge and the easing will accelerate.

It is difficult to attract the attention of young adolescents that the main service using this data is medical. This is because the health issues do not immediately urgent and their interests are relatively low. Conversely, for the youth who enjoy numerous cultural activities, there is merit in the entertainment field. Gene2Me focuses on these popular cultural functions, however, it is still insufficient for entertainment. The more pioneering this field, the more we will use genetic information to make a ripple effect to our users.

The mediators that connect genes and entertainment are diverse. Representatively, there are games, movies, music, and simulations. In particular, VR is a field with potential, interest, and various challenges. VR amplifies the sense of reality and has the advantage that it can be used by fusion with various devices and technologies, so it is suitable for the entertainment field [7].

Celebrity is the most influential factor that can have a ripple effect. An American actress underwent a mastectomy after genetic testing revealed she had an $87 \%$ chance of getting breast cancer. As this incident became a big issue, 23 andMe received the public's attention. Celebrities have recently become more influential due to increased accessibility through SNS. The Ice Bucket Challenge started with the purpose of indirectly experiencing the pain of amyotrophic lateral sclerosis (ALS), also known as Lou Gehrig's disease patients suffering from muscle contraction by flipping ice water. The propagation power of this movement was minimal at first, but when celebrities announced their participation in the movement on social media, it spread exponentially around the world [8]. If entertainment using VR is connected with celebrities, the purpose of this study would be maximized.

The main study is a simulation that combines these factors generated by the genetic information of the celebrity and the users to feel the realistic familiarity with the celebrity. The user can recognize the gap between personalities, what kind of effort is needed to get close to the celebrity, and the real interest of the celebrity through the genetic information of the celebrity.

Besides, by maximizing the intimacy with celebrities with VR, it can induce curiosity and interest in youths who are interested in celebrities, and the fact that celebrities have received genetic information service can have ripple effects. Also, it can be extended to express relationships with interested users through genetic information between users as well as celebrities. A system that checks intimacy by comparing his or her data with a person who has genetic data through AR or MR could also be configured.

\section{B. AR/VR/MR Simulations}

VR has been a hot topic for a few years and the VR industry is developing rapidly. New terms and abbreviations can feel difficult in these situations.
In virtual reality, the user's view is completely blocked and only the virtual world is visible. The "virtual reality" includes the word "reality", but is called "virtual reality" to indicate the meaning of "another reality" that is completely separate from the "reality" we live in. In other words, "reality" and "virtual reality" can be said to be opposite concepts.

In the term "augmented reality", "reality" means the real world we live in. Thus, "augmented reality" means augmenting the real world. It is to use a glass headset that can be seen outside or to project a reality with a mobile phone camera and display additional information on it. Among vehicle navigation, some products display the direction of the vehicle or surrounding buildings on the image received from the camera during operation, which is a representative example of augmented reality.

MR stands for "mixed reality" and "reality" means both real and virtual reality. The mixture of these two realities is called mixed reality. While the concept of AR is to show additional information, in reality, MR can be said to place a virtual object in real space or to recognize a real object and construct a virtual space around it. An example of MR is an application that allows real cars, which are real objects, to be virtually changed.

Fig. 1 depicts the reality-virtuality continuum.

Popular AR/VR/MR devices are illustrated in Fig. 2.

HTC VIVETMis a device for virtual reality games created jointly by SteamVRTM Tracking [10], [11] and head mounted display (HMD) with a $2 * 1080 * 120090 \mathrm{~Hz}$ display. It uses a laser sensor, not a general HMD with a conventional gyro sensor or an infrared camera, and boasts higher freedom and faster tracking speed than the existing HMD. The laser sensor tracks the controller and the HMD in real-time and projects a 360-degree image to the user's eyes. HMD is a device that makes 3D images using binocular parallax. This provides a wide viewing angle, so you can see the virtual reality display even if you move your eyes.

SteamVRTM Tracking library provides libraries for user experience/user interface (UX/UI) using HTC VIVE ${ }^{\mathrm{TM}}$. Through this, it contains the basic information and technology necessary for VR development, such as performing various actions using the camera viewpoint or controller of the protagonist, which should be displayed in VR, which greatly helps to develop with VIVE equipment.

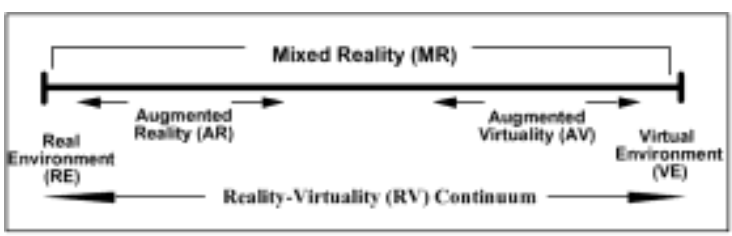

Fig. 1. Reality-virtuality continuum [9]

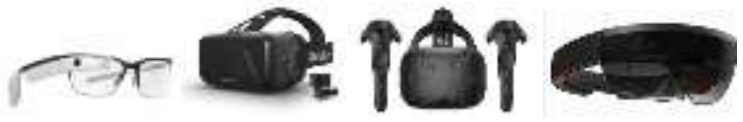

Fig. 2. AR/VR/MR Devices (from Left to Right and Top to Bottom: Google Glasses, Oculus Rift VR Headset, VIVE VR Headset, Microsoft Holo Lens). 
In the case of VR types of equipment, it is easy to overload because it tracks in real-time and runs software. In particular, since the image is made up of 360 degrees, appropriate graphic efficiency is required.

It is a VR Anti-aliasing that helps in this part. Existing antialiasing uses a method of reducing frames of distant elements, but VR is applying this method by changing it for VR.

\section{SYSTEM MODEL}

\section{A. Conceptual Structure of the Simulation System}

For those who want to get acquainted with celebrities and know more, we use HTC VIVETM to create simulations with celebrities. This simulation makes it possible to interact with users based on facts through genetic information of real celebrities rather than virtual celebrities. Through this genetic information, the relationship between the user and the celebrity can be expressed and the constitution and personality can be understood. The basic interaction structure of our simulation system is described in Fig. 3.

After developing the simulation in both AR/VR/MR environments, not only the user dating celebrities but also meeting other users to communicate by interacting between $\mathrm{AR} / \mathrm{VR} / \mathrm{MR}$ environments. It is an extension of the original simulation to influence the genetic information between users.

1) Realization of three-dimensionalization with celebrities in virtual through VR.

2) Realization of visualization through the $3 \mathrm{D}$ engine.

3) Linkage of genetic information to $3 \mathrm{D}$ engine and visualization of data.

Table I summarizes the scopes and their methods to achieve the above three topics of this study.

\section{B. DNA Matching}

Thanks to much researches undergone on DNA sequences pattern matching have been actively undergone [12], [13], it is revealed that particular genes are related to a feature of human personality. C15orf17, message passing interface (MPI), secretory carrier membrane protein 2 (SCAMP2), protein kinase (ULK3), Cytochrome c oxidase subunit 5a (COX5A) are associated with neuroticism [14], Fyn interacts with Nmethyl-D-aspartate (NDMA) receptors and inositol-1,4,5trisphosphate (IP3)-gated channels to regulate calcium influx and intracellular release in the post-synaptic density. It causes an angry temperament [15]. Similarly, a single nucleotide polymorphism, rs322931, is significantly associated with a positive effect [16]. Based on genes that affect human personality, match the conversation choice with the user's genetic information, and match the favorability points of each conversation choice with the celebrity's genetic information.

To match the DNA comparison between the user and the virtual character, a compression algorithm [17] is used. The first step of the compression is encoding edit operation which is replaced, insert and delete. Lempel and Ziv [18], [19] proposed two algorithms to compress data sequences. These algorithms are based on a dictionary that relies on exact repeat. Similarly, a one-pass algorithm based on approximate matching called "Genome Compression" is implemented. The compression gain function based on the optimal prefix is used to evaluate if a particular approximate repeat provides a profit in the encoding.

$$
G(s, t, \lambda)=\max \left\{2|s|-|(|s|, i)|-w_{\lambda} *|\lambda(s, t)|-c, 0\right\}
$$

In the formula above, $\mathrm{s}$ is a prefix of $\mathrm{u}, \mathrm{t}$ is a substring appear at position $\mathrm{i}$ in $\mathrm{v}, 2|\mathrm{~s}|$ is the number of bits we use: 2bits per base $|(|s|, i)|$ is the encoding size of $(|s|, i),|\lambda(s, t)|$ is the number of edit operations in $\lambda(\mathrm{s}, \mathrm{t})$ and $\mathrm{c}$ is the overhead proportional to the size of control bits. The optimal prefix is a prefix $s$ of $u$ when $G(s, t, \lambda)$ is maximized over all $\lambda$ and $t$ while the input is $\mathrm{w}=\mathrm{vu}$. After the parsing and encoding procedure, the optimal prefix search is implemented. The compression is used to find the relatedness between two DNA sequences [17].

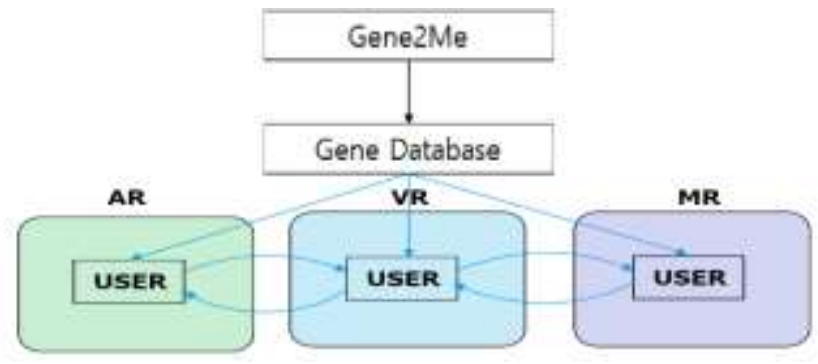

Fig. 3. Interactions between Simulation Environments of the Simulation.

TABLE I. RESEARCH SCOPES AND METHODS

\begin{tabular}{|c|c|}
\hline Scope & Method \\
\hline $\begin{array}{l}\text { Research on } 12 \text { genes } \\
\text { provided by Gene } 2 \mathrm{me}\end{array}$ & $\begin{array}{l}\text { Collect and analyze research data of EDGC } \\
\text { along with existing genetic data. In addition, the } \\
\text { upper limit of the research range should be } \\
\text { found by comparing it with the limited genes. }\end{array}$ \\
\hline $\begin{array}{l}\text { Psychological } \\
\text { interaction study } \\
\text { according to personality } \\
\text { difference between one- } \\
\text { to-one }\end{array}$ & $\begin{array}{l}\text { Study how the other person feels generally } \\
\text { according to the psychology of each individual, } \\
\text { which can be known by genetic information. } \\
\text { Through this, we create a manual that can } \\
\text { organize the relationship by setting the standard } \\
\text { of interaction. }\end{array}$ \\
\hline $\begin{array}{l}\text { Research on genetic } \\
\text { information } \\
\text { databaseization }\end{array}$ & $\begin{array}{l}\text { Organize and use various secondary } \\
\text { information known as genetic information into } \\
\text { data. Besides, the database is made in } \\
\text { consideration of the case of interaction between } \\
\text { secondary information. }\end{array}$ \\
\hline $\begin{array}{l}\text { Research on } \mathrm{AR}, \mathrm{VR} \text {, } \\
\text { and } 360 \text {-degree } \\
\text { rendering of } 3 \mathrm{D} \text { images }\end{array}$ & $\begin{array}{l}\text { Study the function of reading the genetic } \\
\text { information of users through AR, confirming } \\
\text { their genetic relationship, and viewing } \\
\text { individual genetic information. Also, study how } \\
\text { to express the relationship with celebrities } \\
\text { through the AR effect in VR. } \\
\text { We study what effect can be achieved by } \\
\text { linking a 360-degree camera and VR. The } \\
\text { reason for using VR in this study is to } \\
\text { maximize the sense of reality and experience. } \\
\text { This is not simply seen as an image, but } \\
\text { visually makes you feel the most vibrant. }\end{array}$ \\
\hline $\begin{array}{l}\text { Maximizing intimacy } \\
\text { with celebrities }\end{array}$ & $\begin{array}{l}\text { Research how users feel intimacy through the } \\
\text { relationship of genetic information and visual } \\
\text { experiences, despite users do not have } \\
\text { acquaintances with celebrities. Besides, } \\
\text { maximize intimacy are studied by analyzing the } \\
\text { field of celebrity marketing. }\end{array}$ \\
\hline
\end{tabular}




\section{EXPERIMENTS}

Most of the existing studies using genetic information are for medical purposes. The value of genetic information not only for medical purposes but also for entertainment is created through VR. The data of genetic information are specified in detail in a web genetic information database called gene cards. Among the genetic information listed in this GeneCards, the database was reconstructed with the focus on genetic information related to constitution and personality.

When reorganizing the gene database such as Fig. 4, the user, geneInfo, and relation tables were created using Mysql. The user saved the user's genetic information, the geneInfo saved the information of each gene, and the relation saved the correlation of each gene relationship. In this way, the data of gene cards was rearranged and used, and the data was visualized through the GUI of PHPMyAdmin.

It is linked with Unity through SteamVR so that you can see celebrities in a virtual space through HMD VIVE. The viewing angle of the existing VR is about 120 degrees, which is slightly wider than the viewing angle that humans can see, but it has been adjusted to 110 degrees to prevent dizziness. Using 3DMax, a frame was created to visualize and display the data values, and videos taken with a 360-degree camera [21] can be viewed in VR using Unity. Fig. 5 demonstrates a screenshot of our implementation of 360-degree linkage streaming.

When you run the program, a window to select your information appears. Jinwoo Kim, Hun Lim, or Taeho You and will be examples. When you select the name, a window appears where you can choose which celebrity you want to know about. Jinkiskan, Angelina Jolie, etc. are examples. When you select Jinkisukan, Jinkisukan appears in front of you on the VR screen, and the video taken with a 360-degree camera starts. After that, the simulation begins. The correlation with the user through the genetic information required for the simulation is visualized in the background near the celebrity. The following is an example of the simulation process.

If you select Jinkisu Khan, the valiant appearance of Jinkisukhan appears. It gives the feeling of actually talking to the user through VR. After that, a window pops up where you can find out the genetic information about Jinkis Khan's body parts. If you choose an eye, you can get information about the eye and know the protein or food that suits your constitution. And if you click the personality window, you can see the personality created based on the genetic information of Jinkisu Khan. If you click on the relationship, you can compare your personality and see which part is right and which part is not. And a solution comes up as to what kind of effort to make to become close or similar to the celebrity. If you press the home button of the VIVE controller, you will be taken to the screen where you can see your information.

Visual processing is performed so that images taken with a 360 degrees camera can be viewed in VR through the 3D engine Unity, and additional $3 \mathrm{D}$ objects are created using $3 \mathrm{D}$ editors, such as 3D-DOCTOR, 3D MAX, etc. It can be seen in Fig. 5. Also, the object appearance The texture of the image can be viewed more realistically using the image editors, such as Photoshop, etc.

Using many VR engines, such as Unity, Unreal, etc., compatible with SteamVR, you can easily develop UX/UI for using HTC VIVETM, and you can easily design the background through the terrain engine, and asset various anti-aliasing techniques that can prevent overload as much as possible when using VR. Provided as.

Table II shows the development environment that we chose. We use the Unity Engine, an engine that has an advantage in multi-platform interconnection, to interact among different platforms. Also, we decided to use the SteamVR library and HTC VIVETM which have a laser tracking method that has the advantage of less delay time of tracking while moving in a wide space compare to infrared tracking.

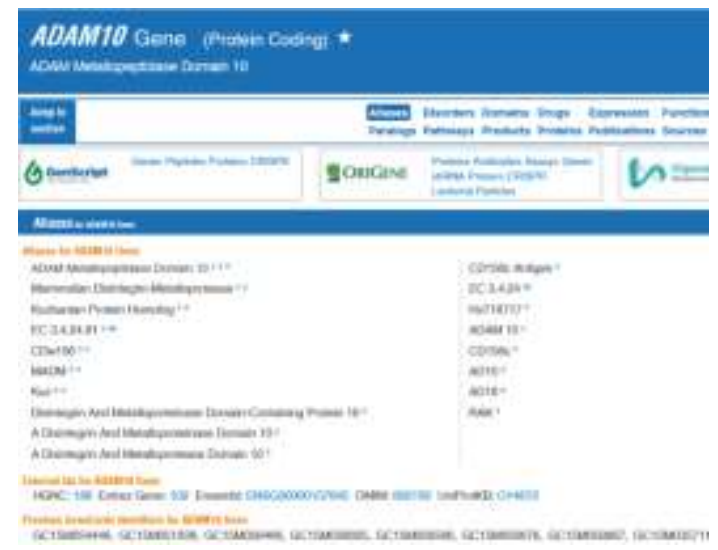

Fig. 4. ADAM10 Genetic Information through GeneCards Database [20].

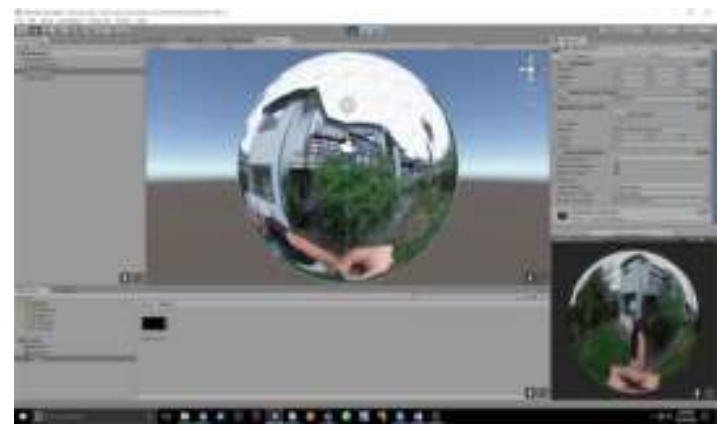

Fig. 5. Screenshot of the Implementation of 360-Degree Linkage Streaming.

TABLE II. SOFTWARE DEVELOPMENT ENVIRONMENTS

\begin{tabular}{|c|l|l|l|}
\hline \multicolumn{2}{|l|}{ 3D Engines } & \multicolumn{2}{l|}{ VR Frameworks } \\
\hline Unity Engine & Unreal Engine & SteamVR & Oculus \\
\hline$\checkmark$ & & $\checkmark$ & \\
\hline
\end{tabular}

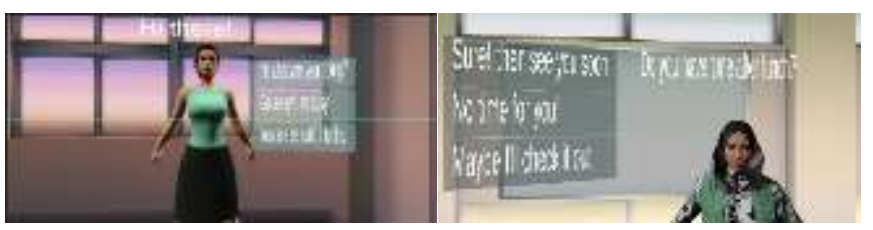

Fig. 6. Examples of an Answer Choice Selection in our Dating Simulation Prototype. 
Based on the genetic information, one selection of the answer choices is created depends on the user's personality. In Fig. 6, due to the user's main personality is anger, the second choice "Go away. I'm busy!" shows the appropriate answer that the user might want to choose. The goal of the game is to gain the most favorable points. The favorability points exist per each choice. Based on the genetic information, the algorithm of determining favorability points is built depending on the celebrity's personality. The system does not tell the user the number of favorability points, instead, the character shows different reactions depends on the result.

In our experiments, participants survey self-personality. The survey determines the genetic information of each participant. play the simulation with two different settings, simulation with random conversation choices and simulation with conversation choices based on the survey. After playing each simulation, record the heart beating, eye tracking position, the favorability point the participants gained, and survey on the satisfaction.

Experiments are performed with the environment of Intel(R) Core(TM) i7-9700 CPU @ 3.00GHz 3.00 GHz, RAM: 16.0GB, GPU RTX 2070 Super, and HTC VIVETM with Dual AMOLED 3.6" diagonal screen, $1080 * 1200$ pixels per eye (2160 × 1200 pixels combined), $90 \mathrm{~Hz}$ refresh rate and 110 degrees of field of view.

\section{DISCUSSION}

We discovered the improvement of human relationships by providing guidelines in the answer choice created based on the genetic information, however, the variety of the human personality is needed to evaluate in detail. To solve this problem, we need to discover more genetic information that influences the human personality. It will solve the limit of selecting the answer in a few options. We only experimented with the virtual characters in virtual reality. It would be meaningful to proceed with the human experiment not only with a virtual character in the virtual environment, but also another human in the virtual environment. Eventually, the result of the experiment between virtual characters and humans should be compared.

To give users more enjoyment, the influence of genes on personality or preference should be more clearly revealed through additional researches. If further researches can provide information that is likely to be preferred according to each individual's genetic information, users can be more interested in and pleased in entertainment based on DNA information.

Besides, it is expected to attract more people's attention through the function of entertainment, not the medical function of the original genetic information. This effect is expected to accelerate the domestic collection of personal genetic information.

\section{CONCLUSIONS}

This paper explains a virtual simulation for entertainment utilizing genetic DNA matching. It also demonstrates that the relationship between celebrities and the user's genetic DNA information, allowing the user to experience a strong affinity with it.
The realistic favorability system based on DNA information can influence the development of human relationships and sociality as well as used as a therapy for people with mental illness. This study contributes that DNA information is related to the formation of human relationships in virtual or real, and it contributed to the discovery that DNA information can contribute to the improvement of interpersonal relationships and mental health. This study also demonstrates the possibility of a new field by utilizing DNA genetic information in the entertainment field using $A R / V R / M R$ technologies.

In future work, we will concentrate on the development of AI agents for helping users to augment the intimacy with a celebrity in the virtual and real world.

\section{ACKNOWLEDGMENT}

This work was supported by Incheon National University Research Grant in 2017 (No. 2017-0320).

\section{REFERENCES}

[1] P. T. Williams. "Quantile-Specific Heritability may Account for GeneEnvironment Interactions Involving Coffee Consumption," Behavior Genetics, Cite this article 50, pp. 119-126, 2020. https://doi.org/10.1007/s10519-019-09989-0.

[2] http://www.23andMe.com (Retrieved September 25, 2020).

[3] https://steamcommunity.com/steamvr (Retrieved September 25, 2020).

[4] R. Plomin, H. M. Chipuer, J. C. Loehlin. "Behavioral genetics and personality," In L. A. Pervin (Ed.), Handbook of personality: Theory and research, pp. 225-243, The Guilford Press, 1990.

[5] http://www.edgc.com (Retrieved September 25, 2020).

[6] http://www.gene2.me (Retrieved September 25, 2020).

[7] M.-L. Champel and S. Lasserre. "The special challenges of offering high quality experience for VR video," Annual Technical Conference and Exhibition, SMPTE 2016, pp. 1-10, 2016. https://doi.org/10.5594/ M001729.

[8] C. W. Chang, P. A. Yang, M. H. Lyu, and K. T. Chuang, "Influential Sustainability on Social Networks," Data Mining (ICDM), 2015 IEEE International Conference on. IEEE, pp. 31-40, 2015. http://dx.doi.org/ 10.1109/ICDM.2015.129.

[9] P. Milgram, “ Milgram, Paul. Some human factors considerations for designing mixed reality interfaces, " Toronto University (Ontario) Dept of Mechanical Engineering, 2006.

[10] J. W. Murray, " Building virtual reality with Unity and Steam VR," CRC Press, 2017.

[11] S. M. LaValle, A. Yershova, M. Katsev, and M. Antonov, "Head tracking for the Oculus Rift," 2014 IEEE International Conference on Robotics and Automation (ICRA). IEEE, pp. 187-194, 2014. https://doi.org/10.1109/ICRA.2014.6906608.

[12] H. T Chang, C. J. Kuo, N. W. Lo, and W. Z. Lv, "DNA sequence representation and comparison based on quaternion number system," International Journal of Advanced Computer Science and Applications (IJACSA), vol. 3, no. 11, 2012. https://doi.org/10.14569/IJACSA. 2012.031107.

[13] Y. Kim, "GenieHD: Efficient DNA pattern matching accelerator using hyperdimensional computing," In: 2020 Design, Automation \& Test in Europe Conference \& Exhibition (DATE), IEEE, pp. 115-120, 2020. https://doi.org/10.23919/DATE48585.2020.9116397.

[14] M. Luciano, J. E. Huffman, A. Arias-Vásquez, A. A. Vinkhuyzen, C. M. Middeldorp, I. Giegling, A. Payton, G. Davies, L. Zgaga, J. Janzing, and $\mathrm{X}$. Ke, "Genome-wide association uncovers shared genetic effects among personality traits and mood states," American Journal of Medical Genetics Part B: Neuropsychiatric Genetics, vol. 159, no. 6, pp. 684695, 2012. https://doi.org/10.1002/ajmg.b.32072.

[15] E. Mick, J. McGough, C. K. Deutsch, J. A. Frazier, D. Kennedy, and R. J. Goldberg, "Genome-wide association study of proneness to anger," 
PloS one, vol.9, no. 1, e87257, 2014. https://doi.org/10.1371/journal. pone. 0087257.

[16] X. Chen,S. Kwong, \& M. Li, "A compression algorithm for DNA sequences and its applications in genome comparison," Genome informatics, 10, pp. 51-61, 1999. https://doi.org/10.1145/332306. 332352.

[17] J. Ziv, \& A. Lempel, A universal algorithm for sequential data compression," IEEE Transactions on information theory, vol. 23, no. 33, pp. 337-343, 1977. https://doi.org/10.1109/TIT.1977.1055714.

[18] Ziv, \& A. Lempel, "Compression of individual sequences via variablerate coding," IEEE transactions on Information Theory, vol. 24, no. 5, pp. 530-536, 1978. https://doi.org/10.1109/TIT.1978.1055934.
[19] M. Safran, I. Bahir, T. Doniger, H. Krug, and A. Sirota-Madi, "GeneCards Version 3: the human gene integrator," Database, 2010. https://doi.org/10.1093/database/baq020.

[20] A. P. Wingo, L. M. Almli, J. S. Stevens, T. Jovanovic, T. S. Wingo, G. Tharp, Y. Li, A. Lori, M. Briscione, P. Jin, and E. B. Binder, "Genomewide association study of positive emotion identifies a genetic variant and a role for microRNAs," Molecular Psychiatry, vol. 22, no. 5, pp. 774-783, 2017.

[21] X. Corbillon,F. De Simone, \& G. Simon, "360-degree video head movement dataset," In Proceedings of the $8^{\text {th }}$ ACM on Multimedia Systems Conference, pp. 199-204, June 2017. 